\title{
ENABLING BUSINESS INTELLIGENCE, KNOWLEDGE MANAGEMENT AND CLINICAL WORKFLOW WITH SINGLEVIEW
}

\author{
Brian J. Kolowitz, University of Pittsburgh Medical Center, bkolowitz@gmail.com \\ Rasu B. Shrestha MD, University of Pittsburgh Medical Center, shrestharb@upmc.edu
}

\begin{abstract}
Healthcare Information Technology (HIT) is changing the face of medicine. Much of the data contained within HIT systems resides in proprietary silos which makes access difficult. SingleView, a patient centric imaging interoperability platform, has made this information more widely available across one large healthcare organization. This has allowed the organization to rethink its HIT strategy in terms of Business Intelligence, Knowledge Management, and Clinical Workflow.
\end{abstract}

Keywords: Health Informatics, Business Intelligence, Knowledge Management, Workflow PACS, Federation, Information Technology

\section{INTRODUCTION}

The ubiquitous proliferation of increasingly powerful Information Technology (IT) has facilitated the transformation of medicine in the United States [1]. Hospitals have rapidly moved towards the implementation of electronic health records, computerized physician order entry system, pharmacy bar coding systems, and the modernization of clinical and research databases [1]. This move is likely, in part, a result of the Institute of Medicine's (IOM) call to action in 2001. The American Recovery and Reinvestment Act substantiated this position while the United States Department of Health and Human Services has identified the meaningful use of Healthcare IT as a key priority [1]. Meaningful Use is intended to help usher in an era of evidence-based medicine and clinical data exchange, where clinical and research data are used to improve caregiver performance, enhance patient safety and allow for the rapid transition from scientific discovery to clinical practice [1].

Clinical data in healthcare continues to be isolated in Information System silos where it is stored in proprietary or incompatible formats [2,3]. Some of this data might be useful for improving care or clinical operations, but many times it is very difficult to access without a time-consuming intermediate process [4]. The National Research Council reported that piecemeal Healthcare IT implementations may fail to realize newer technology promises and may even be counterproductive [5]. Careful implementation of Healthcare IT might transform unwieldy implementations, improve patient outcomes, increase safety, enhance operational efficiency, and support public health efforts [1]. The types of applications that assist in these transformations are commonly known as Business Intelligence (BI) systems [1].

\section{Business Intelligence Systems}

Business Intelligence (BI) systems have traditionally used large volumes of static data that has been extracted, transformed, and loaded into a data warehouse [6]. These systems have helped organizations understand complex processes and relationships by providing a comprehensive view of the organizational data $[7,8]$. Once archived, the data is used to produce reports which can be used for business analytics which may involve process monitoring that can drive business decisions or allow the organization to gain a better understanding of events. BI systems often times provide user notifications when changes in the data occur though e-mail, instant messaging or other electronic means [6]. Applications can be built on top of BI systems and programmed to act on these events triggered by the BI system. These triggers may happen at a predefined time, or in real-time as in Real-Time Business Intelligence (RTBI) systems.

\section{Real-Time Business Intelligence}

The term real-time has many meanings in academic literature and the business world. Some common definitions include: the requirement to obtain zero latency within a process, a process that has access to information whenever 
the information is required, a process that provides information whenever management requires the information, or the ability to derive key performance measures at the point in time the measures are needed [9]. Therefore RealTime Business Intelligence (RTBI) systems provide the same information as traditional BI systems but they do so with zero latency between data collection and user availability [9]. RTBI systems enable the delivery of information, data modeling activities, data analysis, and the execution of actions based on insights in real-time [9].

\section{Healthcare Business Intelligence}

Two distinct understandings of Business Intelligence exist: data-centric and a process centric [10]. The data-centric approach uses BI systems to combine operational data with analytical tools that give decision makers complex and competitive information [10] by improving the timeliness and quality of inputs to the decision process [11] which allows the firm to better understand its own capabilities [12]. The process-centric approach attempts to address shortcomings of the data-centric approach [10] and focuses more on the business processes. It is possible that a large amount of data is left partially or entirely unused in the data-centric approach because the data collection, transformation and integration is isolated from the execution of the business process [13].

Since organizations are a set of well integrated business processes [14], BI should be used to facilitate decision making though integration of information with business processes [10]. This integration is becoming increasingly important in the healthcare sector [10]. Healthcare BI should help management understand the firm's capabilities and facilitate clinical and administrative decision making [10]. Table 1 outlines similarities and differences between healthcare and other business sectors.

Table 1. Comparison of healthcare and other business sectors [15]

\begin{tabular}{|c|c|}
\hline \multicolumn{2}{|r|}{ Differences } \\
\hline Management & $\begin{array}{l}\text { Unified in most sectors, but } \\
\text { healthcare has clinical and } \\
\text { operational reporting. }\end{array}$ \\
\hline Customer & $\begin{array}{l}\text { Clear customer in most sectors, but } \\
\text { healthcare involves variety, } \\
\text { including clinicians, payers, } \\
\text { government, service providers, and } \\
\text { users }\end{array}$ \\
\hline Variants & $\begin{array}{l}\text { Few industries exceed tens of } \\
\text { thousands of variants. Healthcare } \\
\text { typically caters to many tens of } \\
\text { millions of individuals }\end{array}$ \\
\hline $\begin{array}{l}\text { Preference } \\
\text { and choice }\end{array}$ & $\begin{array}{l}\text { Most industrial systems have hard } \\
\text { metrics. In healthcare, people's } \\
\text { feelings and choices matter too }\end{array}$ \\
\hline \multicolumn{2}{|r|}{ Similarities } \\
\hline $\begin{array}{l}\text { Process } \\
\text { orientation }\end{array}$ & $\begin{array}{l}\text { All sectors seek improvement in } \\
\text { quality, cost, and delay through } \\
\text { integrated processes }\end{array}$ \\
\hline $\begin{array}{l}\text { Center of } \\
\text { attention }\end{array}$ & $\begin{array}{l}\text { Healthcare is becoming patient- } \\
\text { centered, following, product- } \\
\text { centered and customer-centered } \\
\text { success in other sectors }\end{array}$ \\
\hline $\begin{array}{l}\text { System } \\
\text { integration }\end{array}$ & $\begin{array}{l}\text { Although healthcare systems are } \\
\text { typically larger; more complex, and } \\
\text { employ more people than other } \\
\text { systems, they still benefit from } \\
\text { whole-system analysis }\end{array}$ \\
\hline
\end{tabular}


Three types of data sources can be found in a healthcare organization: clinical, administrative, and external [10]. Clinical data sources are needed for health service deliver to patients including patient records, medical images, and laboratory result. Administrative data sources are needed to run the healthcare organization and may include personnel or financial data. External data sources may be clinical or business related and are housed by external entities. Examples may include statistical data, medical reports or insurance forms. Healthcare Business Intelligence Systems may need to account for all three types of data sources.

\section{EVALUATION}

In order to support a widespread use of Business Intelligence, a pluggable data layer that allows the dynamic addition and removal of data sources is needed [9]. This technology must include a unified data layer, streamlined development cycle, and automated data mismatch reconciliation [9]. The unified data layer provides a structure that acts as a virtual data warehouse for information systems that require access to the data [9]. The streamlined development cycle is a step-by-step guide for creating meta-data repositories that machines can process as well as providing a mapping across repositories [9]. Automated data mismatch reconciliation is a way of combining and removing mismatches found in the data contained across datasets. The SingleView Framework (Figure 1) satisfies this definition. SingleView virtually unifies data contained in the clinical data sources on-demand when requested. It has a well defined set of steps and standards that must be carried out in order to integrate additional data sources, and can identify mismatches in data contained within the various data stores when the data is requested.

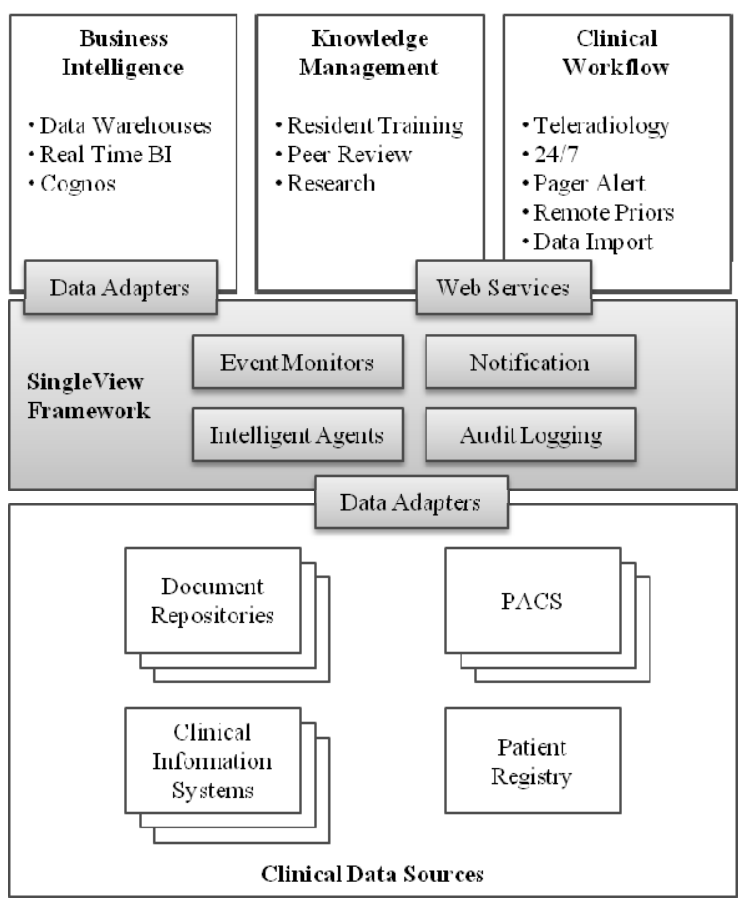

Figure 1. The SingleView Framework

The SingleView framework was initially developed to support clinical workflows around medical imaging. The primary use case was to provide a federated view of 12 independent Picture Archiving and Communication Systems (PACS). In order to do so, a middleware framework was developed to virtually federate the PACS in real-time when the user requested the data.

One model that can be used to evaluate the decision to create SingleView is the Business Pressures-ResponsesSupport model [6]. This model describes how Business Environmental Factors, Organizational Responses, And Decisions and Support interrelate. Business Environmental Factors such as globalization, customer demand, government regulations, and competition create pressures and opportunities for the organization to respond. This 
organizational response can take the form of a new strategy, collaboration with partners, or the development of a new business model among others. The need to respond requires information systems capable of supporting analyses, predictions and decisions. Business Intelligence systems can feed into this decision and support mechanism though the integration of computerized information systems. SingleView has given the organization the ability to integrate information contained within the PACS systems with Business Intelligence systems for the first time.

The model suggests that business and environmental factors pressure the organization as well as create opportunities. Government regulations for securing and sharing medical records and physician demands for a single electronic view of the patient lead to the organization creating a strategy to solve the problem through the development of SingleView. The organizational response has created additional opportunities for business intelligence, analysis, predictions, and integrations.

SingleView was developed as a pluggable framework that allows the rapid addition and removal of components. The SingleView framework has given the organization access to a superset of clinical information previously unattainable within a single application. This has given the organization the opportunity to reevaluate its business processes and develop new framework components in the areas of Business Intelligence, Knowledge Management and Clinical Workflow. The expansion of the SingleView framework components outside its initial use suggests that the information provided is valuable in a multitude of areas across the organization.

In healthcare, organizations require access to real-time information in order to improve care and minimize risks [10]. Some examples of systems that include valuable information but lack the analysis tools necessary to make decisions and improve care included externalizing intelligence in Computerized Provider Order Entry (CPOE) systems, Clinical Decision Support (CDS) applications, and Telemedicine systems [10]. The focus of these systems is not analysis, but the use of analysis makes the systems more valuable [10]. The pluggable SingleView framework can bridge the gap between these systems and business analysis tools. SingleView can and is also being used to support Knowledge Management and other clinical workflow activities beyond its initial design requirements.

\section{SingleView and Business Intelligence}

A major problem in the organization studied is the calculation of Turn-Around-Times (TAT) for radiology reports. The information required to accurately calculate the TAT resides in a number of independent systems including the Radiology Information System (RIS), the Picture Archiving and Communications System (PACS), and a custom internal clinical data repository. Prior to SingleView, calculating the TAT was a time consuming task that did not give the organization confidence in the accuracy of the data. This was primarily due to the reliance on human triggered events.

The SingleView system allows for the creation of pluggable intelligent agents. These agents can interface with the clinical data sources across the enterprise and actively monitor for changes. The intelligent agents contain business logic that captures state transitions and stores the transition along with relevant data from the clinical data sources into a local data-mart like repository. The SingleView framework can then expose the data to the organization's BI system. This has allowed the organization to more accurately monitor the business process and calculate a TAT that more closely represents the actual timing of events than relying on purely human triggered events. In this way SingleView supports Real-Time Business Intelligence within the organization.

\section{SingleView and Knowledge Management}

The organization studied is an academic healthcare organization. One of the responsibilities of the attending physicians is to properly train resident physicians. Additionally, in order to ensure the attending physicians' skills remain up-to-date the various departments engage in daily, weekly and monthly departmental peer reviews.

The organization consists of 12 hospital groups some of which specialize in particular types of medical procedures. Before SingleView, accessing clinical data in another hospital's PACS was a time consuming task. The organization did not have the ability to save and review exam cases across the organization within a single application. 
The SingleView framework has allowed for the creation of an organizational wide Saved Case Manager application. This application allows for the creation of a hierarchical folder structure where exams from any of the independent systems are stored. The physicians can now for the first time sequentially access exams across the independent PACS systems within a single application. Previously these same users would have had to log into and use 12 separate instances of another application. The time to switch between the 12 instances has been eliminated with the introduction of the Saved Case Manager.

The Saved Case Manager application has quickly caught on among PACS users. Radiology administration and technologists can share exams in a quality assurance folder for review across all of the 12 sites. Attending physicians and residents can more easily review rare exam cases from a Saved Case Manager application through the use of an enterprise wide shared folder. In addition, clinical departments that span hospital and PACS silo boundaries can now more easily review cases that span information system silos.

The Saved Case Manager allows the users to customize the hierarchy as well as enter and search on relevant notes regarding the exams. Integration of the Saved Case Manager was designed to fit within the existing workflow of the users. SingleView has allowed the organization to more effectively store, access, and manage data needed to facilitate its Knowledge Management processes though the consolidation of information into a single application.

\section{SingleView and Clinical Workflow Applications}

The increased use of IT to support business operations coupled with Business Intelligence advances have allowed organizations to better understand the way in which various aspects of their business are performing by monitoring and analyzing business processes [16]. Organizations are moving from intra-departmental process evaluation to inter-departmental monitoring and analysis [17]. This can present challenges based on the integration capabilities of the information systems involved. Enterprise Data Warehouses have been a common approach for solving this need because they provide a standard platform for users or systems [16].

As discussed above, the SingleView framework can support Enterprise Data Warehouse aggregation though extraction, transformation, and loading of data from the clinical data sources. The SingleView framework can also be an alternative to the Enterprise Data Warehouses because it can provide much of the same data in real-time without creating a redundant copy. Three examples of applications that support clinical workflows across departmental boundaries using the real-time virtual data warehouse capabilities of SingleView are: SingleView Search, 24-7Teleradiology, and X-Ray Pager Alert.

The SingleView Search provides a federated view of 12 independent Picture Archiving and Communication System (PACS) archives. This allows the physician to view a previously unattainable unified view of the patient regardless of which hospital the patient visited or which PACS the images are stored. SingleView has eliminated the need for the physicians to open separate instances of the PACS application and manually search each system. The SingleView Search tightly integrates into the existing workflows of radiologists and clinicians. As radiologists progress though their un-dictated exam list in their PACS, SingleView proactively searches for and presents relevant prior exams. Similarly, as clinicians view information contained in the patient's electronic medical record, SingleView proactively searches for and presents a comprehensive set the patient's medical image exams. This allows the physicians to eliminate the time consuming and process heavy steps of launching separate PACS application instances and manually searching for exams.

The 24-7 Teleradiology application sits on top of the SingleView framework and includes intelligent agents that monitor emergency rooms and inpatient STAT exams that meet specific clinical criteria. These exams are then presented to an internal Teleradiology team. The team covers 8 hospitals from a centralized location. Their clinical responsibilities involve reading exams that are stored in independent PACS systems.

Teleradiology is a cooperative process that can span organizational departments. The 24-7 intelligent agents in the 24-7 system can direct notifications to specific physicians based upon the type of the exam and business requirement. These business requirements are triggered by events that can occur as a result of internal or external actions. Internal events stem from activities within the company's control [16]. External events are required as part 
of the business process but are outside the control of the organization [16]. The Teleradiology 24-7 system monitors signs of both internal and external events.

The X-Ray Pager Alert system is another application that supports clinical workflow that is built on top of the SingleView framework. This application monitors for events triggered when radiologist provide preliminary interpretations for a specific type of X-Ray exam. Prior to SingleView, the nurses' workflow involved periodically checking the PACS application for these preliminary interpretations. With the advent of SingleView, an intelligent agent was developed to monitor for the preliminary interpretation and notify the nurses. This has allowed the nurse to spend more time treating patients and less time periodically checking the PACS for the existence of the preliminary interpretation.

\section{DISCUSSION}

The Institute of Medicine has identified access to clinical care information across traditional boundaries as being essential to improving the quality of healthcare [18]. The SingleView framework takes a step in this direction by providing a blueprint for how inter-organizational systems may be able to share clinical data while solving an intraorganizational need. SingleView has freed data trapped in silos and given the organization and physicians access to healthcare data that would have been otherwise difficult to retrieve. This ability to access data in and across the silos in a unified platform has been seen as a challenge in healthcare [3], but SingleView has taken significant steps in remedying this problem. The creation of the patient-centric federation platform that allows for a streamlined process of accessing pertinent clinical information across multiple silos of data has in many ways met the IOM's goal. As identified by the IOM, the organization too has noted significant quality of care improvements, whether in the clinical workflow or in the organizational processes.

The information provided by the SingleView platform has given the organization the ability to build and integrate with Business Intelligence systems. This ability is similar to functionality sought after by larger inter-organizational Health Information Exchanges (HIE). Successful Health Information Exchanges have been able to significantly improve reporting of diseases over standard means, monitor disease outbreaks, and facilitate population-level studies [19]. SingleView gives the organization the ability to build similar systems.

Healthcare businesses are activities and work practices within a healthcare organization that are primarily focused on health service delivery [10]. Healthcare processes can require complex routine work that involve a number of departments and people [20]. SingleView not only facilitates the understanding of these processes by integrating data with the BI system, but has allowed the organization to redefine processes utilizing clinical workflow applications.

The SingleView framework has allowed the organization to reevaluate its own business processes. Business processes have two important characteristics: processes have customers and those customers can be internal or external to the organization, and the processes may cross departmental, organizational, cultural, or national boundaries [21]. The SingleView Search, 24-7 application, and X-Ray Pager Alert application demonstrates ways in which SingleView has impacted the organization's healthcare business processes.

The events that SingleView's intelligent monitors use to trigger the actions can be considered Key Indicators (KI). Key Indicators are a set of values that summarize critical business operations [16]. Key Indicators are useful because there can be considerable uncertainty in business processes and business intelligence applications [22]. Organizations may detect problems and trigger decisions based on Key Indicators [16]. The uncertainty is magnified when data comes from multiple sources, stored in different formats, captured by different methods, by different departments or organizations [16]. In the X-Ray Pager Alert case the uncertainty is the timing of the reads performed by the radiologist. SingleView allows the organization to reduce uncertainty by providing a system that monitors and notifies select users when certain actions occur.

The heterogeneity of actors may cause problems when developing and using BI systems because the information may need to be viewed differently across actors [23]. It is crucial to involve the actors when designing the BI system [10]. 


\section{Issues in Information Systems}

Volume XII, No. 1, pp. 70-77, 2011

\section{CONCLUSIONS}

The technical limitations and system boundaries crossed by SingleView are similar but not equal to those that would be encountered by independent organizations. This does not minimize the impact of the system. SingleView has allowed the organization to rethink their Healthcare Information System requirements and trajectory as well as reconsider what is possible in the clinical and academic settings.

SingleView is an interesting case to study because it provides for the opportunity to study the impact of federated PACS silos within a multi-facility geographically dispersed healthcare organization.

This is significant because nearly 25\% Regional Healthcare Information Organizations formed in 2006 were defunct by 2007 [24].

This research is intended to ask the question as to what these technologies mean to healthcare as well as provide an introduction to a few case studies where the impact of the technology can be evaluated in future research. The growth of the framework though the additions of new components in the areas of business intelligence, knowledge management and clinical workflow suggest that the information is useful in these settings. Further research is needed to determine the specific level of usefulness and impact on the users and patient care.

\section{REFERENCES}

1. Ferranti, J.M., et al., Bridging the gap: leveraging business intelligence tools in support of patient safety and financial effectiveness. Journal of the American Medical Informatics Association, 2010. 17(2): p. 136.

2. Fayyad, U. and R. Uthurusamy, Evolving data into mining solutions for insights. Communications of the ACM, 2002. 45(8): p. 31.

3. Hersh, W., Health care information technology: progress and barriers. Jama, 2004. 292(18): p. 2273.

4. Kohavi, R., N.J. Rothleder, and E. Simoudis, Emerging trends in business analytics. Communications of the ACM, 2002. 45(8): p. 45-48.

5. Stead, W.W., et al., Computational technology for effective health care: immediate steps and strategic directions. 2009: National Academies Press.

6. Turban, E., et al., Business intelligence: a managerial approach. 2008: Pearson Prentice Hall.

7. Loshin, D., Business intelligence: getting onboard with emerging IT: the Savvy manager's guide. 2003: Morgan Kaufmann.

8. Liautaud, B., e-Business intelligence: turning information into knowledge into profit. 2000: McGraw-Hill, Inc. New York, NY, USA.

9. Azvine, B., et al. Real time business intelligence for the adaptive enterprise. 2006: IEEE.

10. Mettler, T. and V. Vimarlund, Understanding business intelligence in the context of healthcare. Health Informatics Journal, 2009. 15(3): p. 254.

11. Negash, S., Business Intelligence. Communications of the Association for Information Systems, 2004. 13(1): p. 15.

12. Gluchowski, P., Business Intelligence-Konzepte, Technologien und Einsatzbereiche. HMD-Praxis der Wirtschaftsinformatik, 2001. 222: p. 5-15.

13. Bucher, T. and B. Dinter. Process Orientation of Information Logistics An Empirical Analysis to Assess Benefits, Design Factors, and Realization Approaches. in 41th Annual Hawaii International Conference on System Sciences. 2008. Waikoloa, Big Island, Hawaii: IEEE Computer Society.

14. Hammer, M. and J. Champy, Reengineering the corporation: a manifesto for business revolution. 1993, New York: Harper Collins Publishers.

15. Avison, D. and T. Young, Time to rethink health care and ICT? Communications of the ACM, 2007. 50(6): p. 69-74.

16. Rodriguez, C., et al., Toward Uncertain Business Intelligence: The Case of Key Indicators. Internet Computing, 2010.

17. (2005) GNYHA Members Initiate Clinical Data Exchange Project. Health Care News. 
Volume XII, No. 1, pp. 70-77, 2011

18. Corrigan, J., A. Greiner, and S.M. Erickson, Fostering rapid advances in health care: learning from system demonstrations. 2003: National Academies Press.

19. Shapiro, J.S., Evaluating public health uses of health information exchange. Journal of Biomedical Informatics, 2007. 40(6): p. S46-S49.

20. Vassilacopoulos, G. and E. Paraskevopoulou, A process model basis for evolving hospital information systems. Journal of Medical Systems, 1997. 21(3): p. 141-153.

21. Davenport, T.H. and E. James, The New Industrial Engineering: Information Technology and Business Process Redesign. Sloan Management Review, 1990. 4: p. 11-27.

22. Daniel, F., et al. Managing Data Quality in Business Intelligence Applications. 2008: Citeseer.

23. Checkland, P. and J. Scholes, Soft systems methodology: A 30-year retrospective. 1999: John Wiley.

24. Adler-Milstein, J., et al., The state of regional health information organizations: current activities and financing. Health Affairs, 2008. 27(1): p. w60. 